\title{
ULTRAVIOLET SKY SURVEYS
}

\author{
N. BROSCH \\ Dept. of Astronomy and Astrophysics \\ and the Wise Observatory, Tel Aviv University
}

\section{Introduction}

Among all spectral bands, the ultraviolet has long been neglected, despite the advantage of small space experiments: the sky is very dark, thus detection of faint objects does not compete against an enhanced background (O'Connell 1987) and the telescope construction techniques are very similar (at least longward of $\sim 50 \mathrm{~nm}$ ) to those of optical astronomy.

The short history of UV astronomy can be divided into two eras, until the flight of TD-1 and since the availability of the TD-1 all-sky survey. Very little was accomplished in terms of general sky surveys during this second era. The UV domain may be divided into the "regular" ultraviolet, from shortward of the spectral region observable from ground-based observatories $(\sim 320 \mathrm{~nm})$ to below the Lyman break at $\sim 80 \mathrm{~nm}$, and the region from the Lyman break to the fuzzy beginning of the X-ray domain, arbitrarily defined as $\sim 6 \mathrm{~nm} \approx 200 \mathrm{eV}$ ). The first segment is called "UV" and the second "extreme UV" (EUV). Observational techniques used in the EUV are more similar to those in X-ray astronomy, whereas the UV is more like the optical.

The units used here are "monochromatic magnitudes," defined as:

$$
m(\lambda)=-2.5 \log [f(\lambda)]-21.175
$$

where $f(\lambda)$ is the source flux density in $\mathrm{erg} \mathrm{sec}^{-1} \mathrm{~cm}^{-2} \AA^{-1}$, at wavelength $\lambda$. The background brightness is described in "photon units" (c.u.=count units) which count the photon flux in a spectral band, per $\mathrm{cm}^{2}$, per steradian, and per $\AA$. At $150 \mathrm{~nm}, 1$ c.u. $=1.3210^{-11} \mathrm{erg} \mathrm{cm}{ }^{-2} \mathrm{sec}^{-1} \AA^{-1}$ steradian $^{-1}$, or $1.3210^{-13} \mathrm{~W} \mathrm{~m}^{-2} \mathrm{~nm}^{-1}$ steradian $^{-1}$, or $32.6 \mathrm{mag} \operatorname{arcsec}^{-2}$.

Although only few missions performed full sky surveys in the UV or EUV, many scanned or imaged restricted sky regions and provided information about the deeper UV sky. O'Connell (1991) reviewed UV imaging experiments and their results updated to 1990. 
In parallel with the development of UV astronomy, first steps were taken to study the EUV sky. Detections in the EUV range are hampered by the opacity of the interstellar medium (ISM). From $91.2 \mathrm{~nm}$ shortward to about $10 \mathrm{~nm}$ the opacity is high, because of the photoelectric cross-section of $\mathrm{H}^{0}$, and to a lesser extent of $\mathrm{He}^{0}$ (below $50.4 \mathrm{~nm}$ ) and $\mathrm{He}^{+1}$ (below $22.8 \mathrm{~nm}$ ).

The first studies in the EUV range were with rocket-flown instruments (Henry et al. 1975 a, b, c), which measured a few very bright sources and established calibrators. The earliest observations below Lyman $\alpha$ were by Belyaev et al. (1971). The culmination was the EUV instrument flown on the Apollo-Soyuz mission in 1975, when four EUV point sources were discovered (Lampton et al. 1976, Margon et al. 1976, Haisch et al. 1977, Margon et al. 1978). The Voyager spacecraft explored the EUV sky with their Ultraviolet Spectrometers (UVS: Sandel, Shemansky and Broadfoot 1979). For a number years the two Voyager spacecraft were the most distant astronomical observatories (Holberg 1990, 1991).

\section{The TD-1 Era}

Modern UV astronomy began with the first UV all-sky survey by the ESRO TD-1 satellite, described by Boksenberg et al. (1973). The all-sky catalog of UV sources was published by Thompson et al. (1978) with 31,215 stars with $\mathrm{S} / \mathrm{N}>10$ in all four TD-1 bands. An unpublished version, with lower $\mathrm{S} / \mathrm{N}$, has 58,012 objects. The TD-1 S2/68 experiment is a benchmark against which all other sky surveys are and will be measured.

After TD-1, the various UV and EUV efforts can be characterized as either imagers or spectrometers. Among the imagers, some were orbiters and others were on short-duration flights. Some major missions were ANS and IUE. ANS was described by Van Duinen et al. (1975), Wesselius et al. (1982), and de Boer (1982). One of the greatest successes of any orbiting astronomical instrument was the IUE observatory (Boggess et al. 1978). The IUE data are a valuable resource, mainly after the final reprocessing of all the low-dispersion spectra into the final Uniform Low-Dispersion Archive (ULDA).

The NRL experiment S201 was described by Page et al. (1982) and operated automatically on the Moon during the Apollo 16 mission in April 1972. Ten $20^{\circ}$ diameter fields were observed, the experiment covering $\sim 7 \%$ of the sky. The results were discussed by Carruthers and Page $(1976,1983$, $1984 \mathrm{a}, 1984 \mathrm{~b})$. Two experiments flew on sounding rockets. GUV flew on 21 February 1987 and is described by Onaka et al. (1989). The GUV observations were re-analyzed by Kodaira et al. (1990). The UIT prototype flew on a number of rocket flights and with different focal plane assemblies. Bohlin et al. (1982) described the instrument and its observations of 
the Orion nebula. Other observations are described by Smith and Cornett (1982), Smith et al. (1987), and Bohlin et al. (1990).

UV observations from balloon-borne telescopes at $40+\mathrm{km}$ were performed by a collaboration between the Observatoire de Geneve and the Laboratoire d'Astrophysique Spatiale of Marseille. The stabilized gondola (Huguenin and Magnan 1978) carried telescopes tuned for imaging observations in a bandpass centered at $\sim 200 \mathrm{~nm}$ and $\sim 15 \mathrm{~nm}$ wide. SCAP-2000 was described by Laget (1980), Donas et al. (1981), and Milliard et al. (1983). The results were published by Donas et al. (1987) and Buat et al. (1987, 1989). FOCA is a $39 \mathrm{~cm}$ diameter telescope (Milliard et al. 1991) which surveyed some 70 sq. degrees of the sky to $\sim 19$ mag. Results were reported by Laget et al. (1991a, 1991b), Vuillemin et al. (1991), Courtes et al. (1993), Buat et al. (1994), Bersier et al. (1994), Reichen et al. (1994), Donas et al. (1995), and Petit et al. (1996). Galaxy counts and color distributions for objects in the range 15.0-18.5 mag were published by Milliard et al. (1992) and used to predict UV galaxy counts (Armand and Milliard 1994).

The Wide-Field UV Camera flew in December 1983 on the Space Shuttle and produced some very wide-field UV images (Courtes et al. 1984). The NRL group headed by Carruthers flew a number of far-UV wide-field imagers on rockets (Carruthers et al. 1980). These flights used the Mark II FUVCAM (Carruthers et al. 1993, 1994) which flew on the Space Shuttle in spring 1991. The results were published in a series of papers dealing with individual fields (Schmidt and Carruthers 1993a, 1993b, 1995). The $40 \mathrm{~cm}$ telescope GLAZAR operated on the Mir space station (Tovmassian et al. 1988, 1991a, 1991b) and results were reported by Tovmassian et al. (1993a, 1993b, 1994, 1996a).

FAUST is the Fusee Astronomique pour l'Ultraviolet Spatiale, or the Far Ultraviolet Space Telescope, first described by Deharveng et al. (1979). On SPACELAB-1 in 1983 FAUST did not obtain significant data because of high on-orbit background (Bixler et al. 1984). During the second flight, on board the Shuttle Atlantis in March 1992 (Bowyer et al. 1993), FAUST observed 22 regions $\sim 8^{\circ}$ in diameter and produced a catalog of 4,698 UV sources (Bowyer et al. 1994a). Selected results from the FAUST imagery are by Deharveng et al. (1994), Haikala et al. (1995), and Courtes et al. (1995). A program of systematic investigation of FAUST images takes place the Tel Aviv University and include optical observations from the Wise Observatory. To date, we analyzed completely four FAUST fields, the North Galactic Pole (Brosch et al. 1996a) and three fields covering Virgo (Brosch et al. 1996b).

The Ultraviolet Imaging Telescope (UIT) was described by Stecher et al. (1992). It flew on the Space Shuttle during the ASTRO-1 (December 
1990) and ASTRO-2 flights (March 1995). First results were published in a dedicated publication (1992 Astrophys. J. Lett. 395). Other results are in Hill et al. $(1993,1994,1995 \mathrm{a}, 1995 \mathrm{~b}, 1996)$. The UIT source catalog (Smith et al. 1996) covers 16 sq.degrees of the sky and contains 2244 objects from 48 pointings in the ASTRO-1 flight.

Attempts to measure the diffuse UV background consist of many observations with wide-field instruments. Notable among these are the two Shuttle-borne UVX instruments from JHU and Berkeley (Murthy et al. 1989; Hurwitz et al. 1989). In addition, observations done for other purposes were used to derive the UV background, e.g., Waller et al. (1995).

\section{Modern EUV observations}

The EUV sky was explored by the EUV Wide Field Camera on the ROSAT X-ray all-sky survey satellite described by Pounds and Wells (1991). The first EUV all-sky survey was during 1990-1991 (Pye 1995). Initial results were reported by Pounds et al. (1993) as the WFC Bright Source Catalog (BSC). The reprocessed data make up the 2RE catalog (Pye et al. $1995)$ with 479 EUV sources. In 2 RE $52 \%$ of the sources are active F, G, $\mathrm{K}$, and $\mathrm{M}$ stars, $29 \%$ are hot white dwarfs, and less than $2 \%$ are AGNs.

The region bordering the EUV and the X-rays was explored by the ALEXIS spacecraft (Priedhorsky 1991). The first sky maps were produced on 4 November 1994 . The ALEXIS team calculated that $10 \%$ of the brightest EUVE sources (see below) should be detectable. Most sources are WDs; the catalog from the first three years of operation will probably contain $\leq 50$ sources.

The EUV sky was investigated by the Extreme Ultraviolet Explorer (EUVE) spacecraft (Bowyer and Malina 1991). EUVE mapped the sky in four spectral bands, from 7 to $70 \mathrm{~nm}(18$ to $170 \mathrm{eV})$. The first results were published as "The First EUVE Source Catalog" (Bowyer et al. 1994) with 410 sources. The Second EUVE Source Catalog (2EUVE) has recently been published (Bowyer et al. 1996). The majority of the identified sources in $2 \mathrm{EUVE}(55 \%)$ are $\mathrm{G}, \mathrm{K}$, and $\mathrm{M}$ stars.

A new catalog, to $\sim 60 \%$ of the thresholds of the second EUVE catalog, has been produced by Lampton et al. (1996) with 534 coincident sources between the EUVE $10 \mathrm{~nm}$ list and the ROSAT all-sky survey sources detected in the broadband event window $(0.1-2 \mathrm{keV})$, of which 166 were not previously discovered. Of these, 105 have been identified and $77 \%$ of them are late-type stars. White dwarfs and early-type stars make up only $14 \%$ of the sources, and there are no extragalactic objects at all. 


\section{Comparison of Survey Missions}

The various missions surveying the UV sky can be compared in terms of a "power" parameter $\theta$, introduced by Terebizh (1986), used by Lipovetsky (1992) in a comparison of optical surveys, and slightly modified here:

$$
\theta=\frac{\Omega}{4 \pi} 10^{0.6\left(m_{L}-10\right)}
$$

where $\Omega$ is the sky area covered by the survey $(4 \pi$ for $T D-1)$ and $m_{L}$ is the limiting magnitude of the survey.

The various parameters relevant to the missions discussed here are collected in Tabel 1 . An all-sky survey to $\mathrm{m}_{L} \approx 8.5$ (such as TD-1) has the same "survey power" as one HST WFPC-2 image exposed to show $\mathrm{m}_{U V}=21$ objects. Because of this, and because not all surveys cover the entire sky, it may be more useful to look at another estimator, the density of sources detected (or which are expected to be detected) by a certain experiment. This estimator indicates that the field of UV astronomy retains its vitality; the source density increases exponentially with time.

\section{The Resultant Sky Picture}

The combined results yield a picture in which most of the stars detected by TD-1, FAUST, SCAP and FOCA are early-type B, A and F. However, most of the stars included in the UIT catalog are probably late-type ( $G$ and later). In the FAUST fields where the reduction and identification processes are complete, we find almost equal fractions of A-F stars (70 and 75\%). Except for the fields studied at Tel Aviv (Brosch et al. 1995, 1996), most surveys used exclusively correlations with existing catalogs to identify sources. These sometimes mis-identify objects, as some likely early-type stars are just below the catalog thresholds.

The UV information on galaxies is very sparse and a statistically complete sample of a few 1000's galaxies is lacking. In the absence of very deep surveys in more than a single spectral band, our information about a significant number of galaxies originates from SCAP-2000 (Donas et al. 1987) and FOCA (Milliard et al. 1992). These measurements consist of integrated photometry at $200 \mathrm{~nm}$ of a few hundred galaxies. In the range $16.5-18.5 \mathrm{mag}$ galaxies dominate the source counts at high $|\mathrm{b}|$. These have $\mathrm{B}=18-20$ and $[2000-\mathrm{V}] \approx-1.5$. Using the "field" galaxy luminosity function in the UV from Deharveng et al. (1994) the differential number density is:

$$
\log N(m)=0.625 \times m_{200}-9.5
$$

Studies by UIT and FAUST emphasize the importance of the dust in understanding the UV emission. Bilenko and Brosch (1996) analyzed the 
TABLE 1. UV and EUV survey missions

\begin{tabular}{|c|c|c|c|c|c|c|c|}
\hline Mission & Year & $\Omega$ (ster) & $\mathbf{m}_{L}$ & $\theta$ & $\lambda \lambda(\mathrm{nm})$ & $\mathrm{N}_{\text {sources }}$ & Notes \\
\hline TD-1 & $1968-73$ & $4 \pi$ & 8.8 & 0.19 & $150-280$ & 31,215 & 1 \\
\hline S201 & 1972 & 0.96 & 11 & 0.30 & $125-160$ & 6,266 & \\
\hline WF-UVCAM & 1983 & 1.02 & 9.3 & 0.03 & 193 & ? & \\
\hline SCAP-2000 & 1985 & 1.88 & 13.5 & 18.9 & 200 & 241 & 2 \\
\hline GUV & 1987 & $510^{-3}$ & 14.5 & 0.2 & 156 & 52 & 3 \\
\hline GSFC CAM & $1987+$ & 0.03 & 16.3 & 14.4 & 242 & $\sim 200$ & 4 \\
\hline FOCA & $1990+$ & 0.02 & 19 & 377 & 200 & $\sim 4,000$ & 5 \\
\hline UIT-1 & 1990 & $3.810^{-4}$ & 17 & 0.48 & $\sim 270$ & 2,244 & 6 \\
\hline GLAZAR & 1990 & $4.410^{-3}$ & 8.7 & $610^{-4}$ & 164 & 489 & \\
\hline FUVCAM & 1991 & 0.09 & 10 & $7.510^{-3}$ & 133,178 & 1,252 & 7 \\
\hline FAUST & 1992 & 0.33 & 13.5 & 3.3 & 165 & 4,698 & \\
\hline UIT $1+2$ & 1990,95 & $1.310^{-3}$ & 19 & 26 & $152-270$ & $6,000 ?$ & 8 \\
\hline HST WFPC & $1990+$ & $3.910^{-4}$ & 21 & 123 & $120-300$ & $50,000 ?$ & 9 \\
\hline MSX UVISI & $1997+$ & $4 \pi ?$ & 13.9 & 218 & $180-300$ & ? & \\
\hline GIMI & $1997+$ & $4 \pi$ & 13.6 & 136 & 155 & $2.510^{5}$ & 10 \\
\hline TAUVEX & $1998+$ & 0.06 & 19 & 11,700 & $135-270$ & $10^{6}$ & 11 \\
\hline WFC & $1992 ?$ & $4 \pi$ & - & - & 10,16 & 479 & \\
\hline ALEXIS & $1994+$ & $4 \pi$ & - & - & 13-19 & $50 ?$ & \\
\hline EUVE & $1992 ?$ & $4 \pi$ & - & - & $7-70$ & 734 & 12 \\
\hline
\end{tabular}

Notes to Table 1:

1: The unpublished extended version has 58,012 sources.

2: 92 stars (Laget 1980) and 149 galaxies (Donas et al. 1987).

3: Pointed phase.

4: Virgo observation.

5: Estimated.

6: UIT Catalog.

7: Only the Sag and Sco fields (Shuttle flights) included.

8: Assumes 66 pointings for ASTRO 1 and 100 for ASTRO 2.

9: Assumes 1000 observations with HST with UV filters on WFPC-2.

10: Assumes $2 \times$ stars per magnitude w.r.t. TD-1.

11: Assumes 5000 independent pointings to end-of-life.

12: Number of sources in the 2nd EUVE catalog.

TD-1 catalog and a version of the Hipparcos Input Catalog transformed to the TD- 1 bands and showed that the UV exinction is very patchy, with different extinction gradients on scales $<10^{\circ}$. Tovmassian et al. (1996b) used GLAZAR observations of a 12 degree $^{2}$ area in Crux to establish that the dust distribution is very patchy, with most of the space relatively clear of dust. The EUVE catalogs (Bowyer et al. 1994, 1996; Lampton et al. 
1996) confirm the previously known features of the local ISM (a "tunnel" to CMa with very low HI column density to $200 \mathrm{pc}$ and close to the Galactic plane, a cavity connected with the Gum Nebula in Vela, a shorter $100 \mathrm{pc}$ tunnel to 36 Lynx, and the very clear region in the direction of the Lockman hole).

The accurate measurement of the UV sky background (UVB), with the expectation that it could set meaningful cosmological limits, has been the goal of many rocket, orbital, and deep space experiments. Observational results were summarized by Henry (1982), Bowyer (1990), Bowyer (1991), and Henry (1991). The various origins of the UVB can be separated into "galactic" and "high latitude." The latter is an uniform pedestal, onto which the former is added in various amounts depending on the direction of observation. The "galactic" component can be one order of magnitude more than the "high latitude" one. Most is probably light scattered off dust particles in the ISM and the rest is from the gaseous component of the ISM (HII two photon emission and $\mathrm{H}_{2}$ fluorescense in molecular clouds). The "high latitude" component is also mostly galactic, light scattering off dust clouds at high $|\mathrm{b}|$.

The extragalactic component of the UVB (eUVB) can be at most 100400 c.u. (Murthy and Henry 1995). Whenever $\mathrm{N}(\mathrm{HI})>210^{20} \mathrm{~cm}^{-2}$, the main contributor is dust-scattered starlight. The low level eUVB is probably integrated light of galaxies (Armand et al. 1994), or Milky Way light scattered off dust grains in the Galactic halo (Hurwitz et al. 1991), or intergalactic Ly $\alpha$ clouds contributing their recombination radiation (Henry 1991).

The low UVB away from orbital and galactic contaminants has recently been confirmed by ASTRO-1 UIT images (Waller et al. 1995). After correcting for orbital background and zodiacal light, and after accounting for scattered Galactic light by ISM cirrus clouds (from the IRAS $100 \mu \mathrm{m}$ emission), the extrapolated UV-to-FIR correlation to negligible FIR emission indicates $[\mathrm{eUVB}] \approx 200 \pm 100 \mathrm{c}$.u.

The shorter wavelength UVB has been observed with the Voyager UVS down to $50 \mathrm{~nm}$ (Holberg 1986). A very deep EUVE spectroscopic observation of a large region on the ecliptic has recently been reported (Jelinsky et al. 1995) but the only emission lines observed were He I and He II (58.4, 53.7 , and $30.4 \mathrm{~nm}$ ), which originate from scatted Sunlight by the geocoronal and/or interplanetary medium and no continuum was detected.

The "true" eUVB can be evaluated from the FOCA data (Milliard et al. 1992). The galaxy counts, for $15.0 \geq m_{200} \geq 18.5$, extrapolated to $\mathrm{m}_{200}=20.0$, give a contribution of $\sim 100$ c.u.'s from only UV galaxies. Milliard (1996, private communication) studied the nature of sources in the 
A2111 FOCA field. The majority are emission-line galaxies, only $16 \%$ in $\mathrm{A} 2111$ and the rest are in the foreground or background up to $\mathrm{z} \approx 0.7$.

The detection and identification of faint UV galaxies may help resolve the issue of a real eUVB. Recent indications are that the eUVB is negligible. Waller et al. (1995) estimated $200 \pm 100 \mathrm{c.u}$. in the UIT near-UV band. Unpublished results from an analysis of 17 years of Voyager UVS spectra (Murthy et al. 1996, in preparation) indicate that at $\sim 100 \mathrm{~nm}$ the UVB is $\leq 100$ c.u. $(1 \sigma)$. However, when adopting the FOCA galaxy counts and extrapolating to $\mathrm{m}_{U V}=20$ and to $\sim 1000 \AA$ using the SEDs of starburst galaxies from Kinney et al. (1996), the contribution due to sources unresolved by UVS violates the Murthy et al. limit. An extrapolation to $\mathrm{m}_{U V}=23$ violates also the UIT constraint. It is therefore necessary to investigate the faint end of the UV galaxy distribution to understand the nature and reality of the eUVB.

\section{The Future}

Two UV missions are approved, funded, built, and integrated into their carrier spacecraft. These are the UVISI on MSX, and GIMI on ARGUS, which will produce full or partial UV sky surveys. MSX was launched on 24 April 1996 and the operation of UVISI is expected to start in 1997. ARGUS has been slightly delayed and will be launched in 1997 .

The narrow field UV imager of UVISI (Heffernan et al. 1996) is sensitive to sources which produce 2 photons $\mathrm{cm}^{-2} \mathrm{sec}^{-1}$, i.e., $\mathrm{m}_{L} \approx 13.9$ (monochromatic, at $240 \mathrm{~nm}$ ). It is not clear how much of the sky will MSX survey. However, GIMI on ARGOS has as a declared goal the production of a full sky survey in three UV bands. The most recent description of GIMI (Carruthers and Seeley 1996) indicates $\mathrm{m}_{L} \approx 13.6$.

TAUVEX, Tel Aviv University UV Explorer, (Brosch et al. 1994) is the most advanced attempt to design, build and operate a flexible instrument for observations in the entire UV band. TAUVEX images the same 0.9 FOV with three co-aligned telescopes with an image quality of about $10^{\prime \prime}$. It is part of the scientific complement of the Spectrum X- $\gamma$ spacecraft. The projected performance is detection of objects $19 \mathrm{mag}$ and brighter with $\mathrm{S} / \mathrm{N}>10$ in three bands $\sim 40 \mathrm{~nm}$ wide, after a four hour pointing. At high $|\mathrm{b}|$, each pointing is expected to result in the detection of some tens of QSOs and AGNs (mainly low-z objects) and some hundreds of galaxies and stars. A three-year operation will cover $\sim 5 \%$ of the sky to $\mathrm{m}_{U V} \approx 19 \mathrm{mag}$.

The deepest observations of the UV sky should be made far from the Earth's geocorona, away from the Sun, and out of the ecliptic. Multipurpose missions to the outer planets could be used for astronomy during their cruise phase. Much cheaper options are UV observations from long- 
duration super-pressure balloons at $40-45 \mathrm{~km}$ altitude, for flights of weeks to months, with real-time operation using the TDRSS data relay satellites (Israel 1993). The reactivation of the GLAZAR telescope should be considered. An interesting possibility is to conduct a deep UV survey of a very small fraction of the sky with the HST, to learn about the faintest UV sources, beyond the capability of UIT, FOCA, or TAUVEX.

\section{Conclusions}

A review of the UV and EUV sky reveals that while most UV sources are early-type stars, most EUV sources are late-type stars. It is clear that UV and EUV observations are hampered by ISM opacity. Very faint UV sources are mostly galaxies, and the UVB, if it exists, is very faint and may be fully explained by galaxies.

Significant improvement in our knowledge of the UV sky can be achieved through judicious use of multi-purpose platforms, where the UV science piggy-backs on other spectral ranges. The heritage of past missions should be fully realized before embarking on new adventures. In this light, the extension of the EUVE mission in a low-cost mode is commendable; the extension of the right-angle surveys should bring in more faint EUV sources.

What science needs is either a multi-spectral all-sky UV imaging survey to 19-20 monochromatic magnitude, or a combination of cheaper alternatives, including a long-duration, very high-altitude balloon with a FOCAtype telescope and with a high-resolution detector with electronic readout for a sky survey in the $200 \mathrm{~nm}$ band, combined with the reactivation of the GLAZAR telescope after the status of its optics has been reassessed, with a modern, digital, photon-counting detector, and a mini-survey with HST in the UV. Additionally, an extended EUV all-sky survey, $\sim 100 \times$ more sensitive than EUVE and with better angular resolution, is still required.

\section{Acknowledgements}

UV research at Tel Aviv University is supported by special grants from the Israel Space Agency, from the Austrian Friends of Tel Aviv University, and from a Center of Excellence Award from the Israel Academy of Sciences. I acknowledge support from a US-Israel Binational Award to study FAUST UV sources, and the hospitality of NORDITA and the Danish Space Research Institute where parts of this review were prepared. I am grateful for the help of David Bersier, Benny Bilenko, Jeff Bloch, Jeff Hill, David Israel, Michael Lampton, and Bruno Milliard in preparing this review. 


\section{References}

Armand, C. and Milliard, B. 1994 Astron. Astrophys. 282, 1

Belyaev, V.P. et al. 1971 Cosmic Res. 8, 677

Bersier, D. et al. 1994 Astron. Astrophys. 286, 37

Bilenko, B. and Brosch, N. 1996 preprint

Bixler, J. et al. 1984 Science 225, 184

Boggess, A. et al. 1978 Nature 275, 372

Bohlin, R.C. et al. 1982 Astrophys. J. 255, 87

Bohlin, R.C. et al. 1990 Astrophys. J. 352, 55

Boksenberg, A. et al. 1973 Mon. Not. R. astr. Soc. 163, 291

Bowyer, S. 1990 in "The Galactic and Extragalactic Background Radiation" (S. Bowyer and Ch. Leinert, eds.), Dordrecht: Reidel, p. 171

Bowyer, S. 1991 Ann. Rev. Astron. Astrophys. 29, 59

Bowyer, S. and Malina, R.F. 1991 "Extreme Ultraviolet Astronomy" (R.F.Malina and S. Bowyer, eds.), New York: Pergamon Press, p. 397

Bowyer, S. et al. 1993 Astrophys. J. 415, 875

Bowyer, S. et al. 1994a Astrophys. J. Suppl. 96, 461

Bowyer, S. et al. 1994 Astrophys. J. Suppl. 93, 569

Bowyer, S. et al. 1996 Astrophys. J. Suppl. 102, 129

Brosch, N. 1991 Mon. Not. R. astr. Soc. 250, 780

Brosch, N. et al. 1994 SPIE 2279, p. 469

Brosch, N. et al. 1995 Astrophys. J. 450, 137

Brosch, N. et al. 1996 preprint

Buat, V. et al. 1987 Astron. Astrophys. 185, 33

Buat, V. et al. 1989 Astron. Astrophys. 223, 42

Buat, V. et al. 1994 Astron. Astrophys. 281, 666

Carruthers, G.R. et al. 1980 Astrophys. J. 237, 438

Carruthers, G. et al. 1993 SPIE 1764, p. 21

Carruthers, G. et al. 1994 SPIE 2282, p. 184

Carruthers, G.R. and Page, T. 1976 Astrophys. J. 205, 397

Carruthers, G.R. and Page, T. 1983 Astrophys. J. Suppl. 53, 623

Carruthers, G.R. and Page, T. 1984a Astrophys. J. Suppl. 54, 271

Carruthers, G.R. and Page, T. 1984b Astrophys. J. Suppl. 55, 101

Carruthers, G.R. and Seeley, T.D. 1996 SPIE meeting 2831, Denver: August 7-8, 1996

Courtes, G. et al. 1984 Science 225, 179

Courtes, G. et al. 1993 Astron. Astrophys. 268, 419

Courtes, G. et al. 1995 Astron. Astrophys. 297, 338

Deharveng, J.-M. et al. 1979 Space Science Instrumentation 5, 21

Deharveng, J.-M. et al. 1994, Astron. Astrophys. 289, 715

Donas, J. et al. 1987, Astron. Astrophys. 180, 12

Donas, J. et al. 1981 Astron. Astrophys. 97, L7

Donas, J. et al. 1995 Astron. Astrophys. 303, 661

Haikala, L.K. et al. Astrophys. J. Lett. 443, L33

Haisch, B. et al. 1976 Astrophys. J. 213, L119

Heffernan, K.J. et al. 1996 Johns Hopkins APL Technical Digest 17, 198

Henry, P. et al. 1975a Astrophys. J. 195, 107

Henry, P. et al. 1975b Rev. Sci. Instr. 46, 355

Henry, P. et al. 1975c Astrophys. J. Lett. 197, L117

Henry, R.C. 1982 10-th Texas Symposium, Ann NY Acad. Sci., p. 428

Henry, R.C. 1991 Ann. Rev. Astron. Astrophys. 29, 89

Hill, J.K. et al. 1993 Astrophys. J. 413, 604

Hill, J.K. et al. 1994 Astrophys. J. 425, 122

Hill, J.K. et al. 1995a Astrophys. J. Suppl. 98, 595

Hill, J.K. et al. 1995b Astrophys. J. 446, 622 
Hill, J.K. et al. 1996 Astrophys. J. Lett. preprint

Holberg, J.B. 1990 in "Observatories in Earth Orbit and Beyond," p. 49

Holberg, J.B. 1991 in "Extreme Ultraviolet Astronomy," p.8

Huguenin, D. and Magnan, A. 1978 ESA SP-135, 403

Hurwitz, M. et al. 1991 Astrophys. J. 372, 167

Israel, D.J. 1993 IEEE AES Systems Magazine (Feb. 1993), p. 43

Jelinsky, P. et al. 1995 Astrophys. J. 442, 653

Kinney, A.L. et al. 1996 Astrophys. J. 467, 38

Kodaira, K. et al. 1990 Astrophys. J. 363, 422

Laget, M. 1980 Astron. Astrophys. 81, 37

Laget, M. et al. 1991a Adv. Space Res. 11, 139

Laget, M. et al. 1991b Astron. Astrophys. 259, 510

Lampton, M. et al. 1976 Astrophys. J. Lett. 203, L71

Lampton, M. et al. 1996 preprint

Lipovetsky, V.A. 1992 in "Astronomy from Wide-Field Imaging" (H.T MacGillivray et al. eds.), Dordrecht: Reidel, p. 3

Margon, B. et al. 1976 Astrophys. J. Lett. 210, L79

Margon, B. et al. 1978 Astrophys. J. 224, 167

Milliard, B. et al. 1983 ESA SP-183, p. 409

Milliard, B. et al. 1991 Adv. Space Res. 11, 135

Milliard, B. et al. 1992 Astron. Astrophys. 257, 24

Murthy, J. et al. 1989 Astrophys. J. 336, 954

Murthy, J. and Henry, R.C. 1995 Astrophys. J. 448, 848

O'Connell, R.W. 1987 Astron. J. 94, 876

O'Connell, R.W. 1991 Adv. Space Res. 11, 71

Onaka, T. et al. 1989 Astrophys. J. 342, 235

Page, T. et al. 1982 NRL Report 8487

Petit, H. et al. 1996 Astron. Astrophys. 309, 446

Pounds, K. and Wells, A.A. 1991 Adv. Space Res. 11, 125

Pounds, K.A. et al. 1993 Mon. Not. R. astr. Soc. 260, 77

Priedhorsky, W. et al. 1991 in "Extreme Ultraviolet Astronomy," p. 464

Pye, J.P. 1995 in "Astrophysics in the Extreme Ultraviolet" proceedings of IAU Colloquium no. 152

Pye, J.P. et al. 1995 Mon. Not. R. astr. Soc. 274, 1165

Reichen, M. et al. 1994 Astron. Astrophys. Suppl. 106, 523

Sandel, B.R. et al. 1979, Astrophys. J. 227, 808

Schmidt, E.G. and Carruthers, G.R. 1993a Astrophys. J. 408, 484

Schmidt, E.G. and Carruthers, G.R. 1993b Astrophys. J. Suppl. 89, 259

Schmidt, E.G. and Carruthers, G.R. 1995 Astrophys. J. Suppl. 96, 605

Smith, A.M. and Cornett, R.H. 1982 Astrophys. J. 261, 1

Smith, A.M. et al. 1987 Astrophys. J. 320, 609

Smith, E.P. et al. 1996 Astrophys. J. Suppl. 104, 287

Terebizh, V.Yu. 1986 Contributions of Special Astrophys. Obs. 50, 79

Thompson, G.I. et al. 1978 "Catalog of Stellar Ultraviolet Fluxes," SRC

Tovmassian, H.M. et al. 1988 Pis'ma v Astron. Zh. 14, 291 (Sov. Astron. Lett. 14, 123)

Tovmassian, H.M. et al. 1991a in "Astronomy from Wide-Field Imaging," p. 55

Tovmassian, H.T. et al. 1991b in "Astronomy from Wide-Field Imaging," p. 473

Tovmassian, H.M. et al. 1994 Astrophys. Space Sci. 213, 175

Tovmassian, H.M. et al. 1993b Astron. Astrophys. Suppl. 100, 501

Tovmassian, H.M. et al. 1993a Astron. J. 106, 627

Tovmassian, H.M. et al. 1996a Astron. J. 111, 299

Tovmassian, H.M. et al. 1996b Astron. J. 111, 306

Van Duinen, R.J. et al. 1975 Astron. Astrophys. 39, 159

Vuillemin, A. et al. 1991 Adv. Space Res. 11, 143

Waller, W.H. et al. 1995 Astron. J. 110, 1255 